\title{
Using short-term measures of behaviour to estimate long-term fitness of southern elephant seals
}

L. F. New ${ }^{1,2,3, *}$, J. S. Clark ${ }^{4}$, D. P. Costa ${ }^{5}$, E. Fleishman ${ }^{6}$, M. A. Hindell ${ }^{7}$, T. Klanjš ek $^{8}$, D. Lusseau', S. Kraus ${ }^{10}$, C. R. McMahon ${ }^{7,11}$, P. W. Robinson ${ }^{5}$, R. S. Schick ${ }^{3,4}$, L. K. Schwarz ${ }^{5}$, S. E. Simmons ${ }^{1}$, L. Thomas ${ }^{2,3}$, P. Tyack ${ }^{2}$, J. Harwood ${ }^{2,3}$

${ }^{1}$ Marine Mammal Commission, Bethesda, Maryland 20814, USA

${ }^{2}$ Scottish Oceans Institute, University of St Andrews, St Andrews KY16 8LB, UK

${ }^{3}$ Centre for Research into Ecological and Environmental Modelling, University of St Andrews, St Andrews KY16 9LZ, UK

${ }^{4}$ Nicholas School of the Environment, Duke University, Durham, North Carolina 27708, USA

${ }^{5}$ Department of Ecology and Evolutionary Biology, University of California, Santa Cruz, California 95060, USA

${ }^{6} \mathrm{John}$ Muir Institute of the Environment, University of California, Davis, California 95616, USA

${ }^{7}$ Institute for Marine and Antarctic Studies, University of Tasmania, Hobart, TAS 7001, Australia

${ }^{8}$ Ruðer Boškovič Institute, 10000 Zagreb, Croatia

${ }^{9}$ Institute of Biological and Environmental Sciences and Marine Alliance Science and Technology for Scotland,

University of Aberdeen, Aberdeen AB24 2TZ, UK

${ }^{10}$ New England Aquarium, Boston, Massachusetts 02110, USA

${ }^{11}$ Research Institute for the Environment and Livelihoods, Charles Darwin University, Darwin, NT 0909, Australia

*Corresponding author: lnew@usgs.gov

Marine Ecology Progress Series 496: 99-108 (2014)

Supplement. Figures assessing the model fit for all 30 analysed post-moult foraging trips 


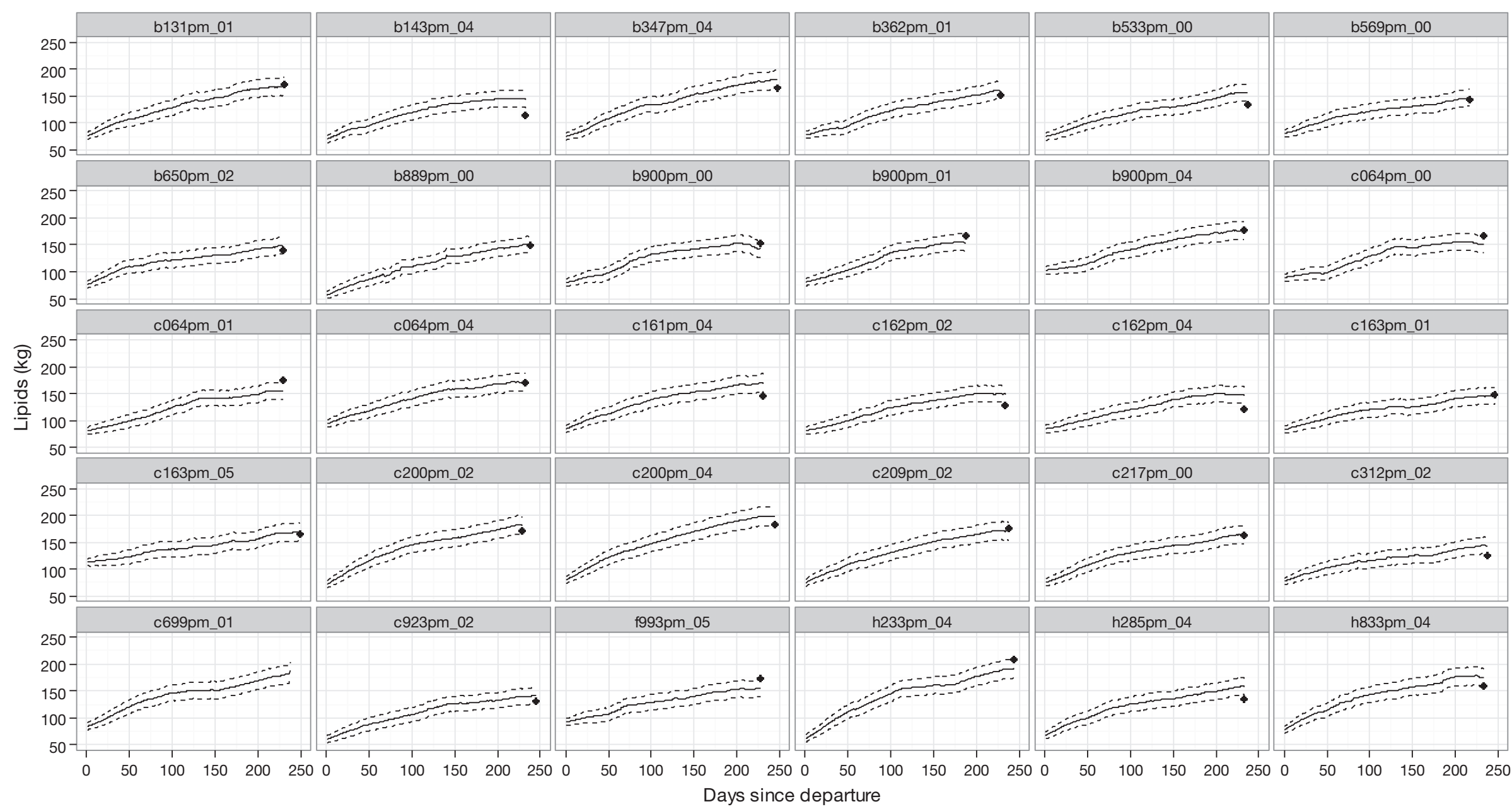

Fig. S1. Mirounga leonina. Changes over time in the estimated lipid mass during 30 post-moult foraging trips of female southern elephant seals from Macquarie Island, Australia (solid line), with 95\% confidence intervals (dashed lines). The black dot indicates the measured final lipid mass of the seal upon return to Macquarie Island. The final lipid mass for seal c699pm_01 is not shown, since the inclusion of this value $(>250 \mathrm{~kg})$ inhibits the clarity of the figure 